\title{
Assessment of glycan interactions of clinical and avian isolates of Campylobacter jejuni
}

\author{
Christopher J Day, Greg Tram, Lauren E Hartley-Tassell, Joe Tiralongo and Victoria Korolik*
}

\begin{abstract}
Background: Campylobacter jejuni strain 11168 was demonstrated to have a broad specificity for eukaryotic surface glycosylation using glycan array analysis. The initial screen indicated that sialic acid and mannose are important binding partners after environmental stress, while galactose and fucose structures are likely to be involved in persistent infection.

Results: In this broader study, five additional human/clinical isolates and six chicken isolates were fully assessed to determine their glycan binding capacity using an extended glycan array. C. jejuni 11168 was rescreened here due to the presence of glycoaminoglycan (GAG) and other structures that were not available on our previous glycan array. The current array analysis of additional C. jejuni strains confirmed the growth condition dependent differences in glycan binding that was previously observed for $C$. jejuni 11168. We noted strain to strain variations, particularly for the human isolates C. jejuni 520 and 81116 and the chicken isolate $C$. jejuni 331, with the majority of differences observed in galactose, mannose and GAG binding. Chicken isolates were found to bind to a broader range of glycans compared to the human isolates, recognising branched mannose and carageenan (red seaweed) glycans. Glycan array data was confirmed using cell-based lectin inhibition assays with the fucose (UEA-I) and mannose (ConA) binding lectins.

Conclusions: This study confirms that all $C$. jejuni strains tested bind to a broad range of glycans, with the majority of strains (all except 81116) altering recognition of sialic acid and mannose after environmental stress. Galactose and fucose structures were bound best by all strains when C. jejuni was grown under host like conditions confirming the likelihood of these structures being involved in persistent infection.
\end{abstract}

Keywords: Host-bacterial interactions, Glycan array, Glycoconjugates

\section{Background}

Glycan or carbohydrate based host-bacterial interactions are crucial for the initiation of both disease and colonisation of many bacteria species [1-4]. Specifically, the ability to recognise a broad range of host cell surface glycosylation has been shown to be crucial for the adherence and infectivity of C. jejuni [3,4]. In vivo, fucosylated glycans present on human breast milk proteins and free fucosylated oligosaccharides can reduce the incidence of $C$. jejuni infections in breastfeeding infants $[5,6]$. While in vitro, blocking the surface glycans with lectins to fucosylated and terminal galactose structures can completely inhibit the adherence of C. jejuni to Caco-2 cells [3].

\footnotetext{
* Correspondence: v.korolik@griffith.edu.au

Institute for Glycomics, G26, Griffith University Gold Coast Campus, Queensland 4222, Australia
}

Glycan array analysis of $C$. jejuni 11168 found that binding of $C$. jejuni to mannosylated and sialylated glycoconjugates was dependent on the growth or maintenance conditions of the bacteria [3]. After exposure of $C$. jejuni to environmental stress (normal oxygen and room temperature) the bacteria were found to bind extensively to mannosylated and sialylated glycoconjugates. This binding was eliminated when the bacteria were grown under microaerobic conditions at either $37^{\circ} \mathrm{C}$ or $42^{\circ} \mathrm{C}$; at these conditions binding to galactose and fucose predominated [3].

Within the Epsilon proteobacteria a complete spectrum of glycans involved in host bacterial interactions has been determined for Helicobacter pylori. Like C. jejuni, H. pylori exhibits broad complexity in carbohydrate-binding specificities. It has been proposed for H. pylori that initial interactions with host tissues may be achieved through binding to the normal gastric epithelium which expresses non- 
sialylated glycoconjugates such as the Lewis B antigen through the action of the lectin BabA $[2,7,8]$. In addition, persistence of $H$. pylori infection appears to be mediated through the binding of the lectin SabA to the sialylated diseased epithelium of the chronically infected stomach $[2,8,9]$. In contrast, the initial interactions for $C$. jejuni 11168, appear to be to highly sialylated and mannosylated structures such as those found on human glycoprotein MUC1, abundant in human intestinal mucosa $[3,4,8,10]$. While persistent C. jejuni infection in crypts of the intestinal epithelium seems to depend on fucose and galactose, structures more readily found on the gel forming mucins such as MUC2 $[3,4,8]$.

C. jejuni has a broad host range, infecting a wide range of both avian and mammalian hosts. Within eukaryotes there are numerous differences between the types of surface glycans present with differences in sialic acids (Neu5Ac/ Neu5Gc) expressed, level and linkages type to fucose and the degree of terminal $\alpha / \beta$ linkages to terminal galactose residues [11-14]. C. jejuni can be either infectious or commensal in different hosts, with disease typically observed in mammals and commensal relationships with avian species. Whether or not the host temperature or glycan expression may play a role in this is still to be elucidated. In this study we analysed the glycan binding profile of twelve strains of $C$. jejuni isolated from human and avian hosts with differing invasive profiles to determine if there are any glycan binding differences between invasive and non-invasive $C$. jejuni.

\section{Results}

\section{Glycan array analysis of $C$. jejuni strains}

Glycan array screening was performed on $C$. jejuni strains grown under different conditions to determine the glycan binding specificity for each strain tested. Each of the twelve C. jejuni strains was found to recognise a large variety of glycoconjugates present on the array (Tables 1, 2, 3 and 4; see Additional file 1: Table S1 for full list and structures of glycans).

All but two of the strains, C. jejuni 331 and 520, bound all galactose structures present on the array (Table 1). The chicken isolate $C$. jejuni 331 recognised the least number of terminal galactose structures only recognising 15 of the 24 printed structures. Of the nine terminal galactose structures that $C$. jejuni 331 fails to recognise, seven are disaccharides and no binding was observed to disaccharides containing GalNAc residues. Human isolate $C$. jejuni 520 failed to bind two structures; one was asialo-GM1 $(1 \mathrm{~F})$ and a terminal $\alpha-1-4$ linked galactose $(1 \mathrm{~K})$, both these structures offer unique terminal glycans, with no other glycan present on the array presenting the same structure on the reducing end (Table 1).

Most variability was observed in binding to $\mathrm{N}$ acetylglucosamine (Table 2; 4A-4E), mannosylated (Table 2; $5 \mathrm{~A}-5 \mathrm{H}$ ) and sialylated (Table 3; 10A-11D) glycans, with different strains recognising variable subsets of each of these structures. Binding to mannose and sialic acid was consistently growth condition dependent for the majority of strains tested (10/12) with differential binding occurring depending on whether the strains were grown under conditions mimicking different hosts $\left(37 / 42^{\circ} \mathrm{C}\right.$ with microaerobic conditions) or environmentally exposed (room temperature with normal oxygen; Tables 2 and 3).

Chitin structures ( $\mathrm{GlcNAc}_{\mathrm{n}}$; Table 2, 4A-4D) are present on the array as a variable repeat length glycan (2-5 sugars in length), with the recognition of these repeat lengths differing between strains tested. The non-invasive chicken isolate 331 has a preference for the smaller repeats $\left(\mathrm{GlCNAc}_{2-3}\right.$; Table 2, 4A and B), while almost all other strains preferentially bound to the larger fragments (GlcNAc 5 ; Table 2, 4D). C. jejuni 11168 was found not to bind any of these structures.

Though sialic acid was in general only recognised under conditions mimicking environmental stress there were several sialylated structures that were also recognised by all $C$. jejuni strains grow under host-like conditions. Typically the sialylated structures recognised by $C$. jejuni grown under host-like conditions were also fucosylated. The most noteworthy was binding of the sialylated and fucosylated structures, SialylLewis A and X (Table 3, 10A and B). Binding differences were observed for human isolates 351,375 and 520 and chicken isolates 331, 434 and 506, however, these differences could not be attributed to a specific host, chicken or human. Also, C. jejuni strains 520 (human), 81116 (human) and 019 (chicken) were shown to bind at least one non-fucoslylated sialic acid containing structure when grown under host-like conditions. For $C$. jejuni 520 and 019 this structure is a complex, branched, $N$-linked glycan that contains within its 11 residues; a mixture of sialic acid (terminal positions on the branches), galactose, mannose and glucosamine linked directly to an asparagine. Therefore, the binding of sialic acid by $C$. jejuni 520 and 019 to this structure may not be due to any specific recognition of sialic acid under host-like growth conditions.

All C. jejuni strains widely recognised structures containing fucose including the bianternary structure present in the sialylated glycans (Table 3; 10D), with no significant difference observed between the twelve strains (data not shown; see Additional file 1: Table S1 for list of structures tested).

Numerous differences were observed for the binding of glycoaminoglycans (GAGs) and related structures between the C. jejuni strains tested (Table 4). Recognition of GAG structures has not previously been reported for C. jejuni. We found that carageenan structures (red seaweed extract with structural similarities to GAGs) were preferred by chicken isolates, with five of the six isolates recognising these structures. Only $C$. jejuni 331 did not bind to these structures (Table 4; 12A-F). Of the human 
Table 1 Terminal galactose binding from the glycan array analysis of twelve $C$. jejuni strains

\begin{tabular}{|c|c|c|c|c|c|c|c|c|c|c|c|c|c|c|c|c|c|c|c|c|c|c|c|c|c|c|c|c|c|c|c|c|c|c|c|c|}
\hline \multirow[t]{3}{*}{ Glycan ID } & \multicolumn{18}{|c|}{ Human } & \multicolumn{18}{|c|}{ Chicken } \\
\hline & \multicolumn{3}{|c|}{11168} & \multicolumn{3}{|c|}{351} & \multicolumn{3}{|c|}{375} & \multicolumn{3}{|c|}{520} & \multicolumn{3}{|c|}{81116} & \multicolumn{3}{|c|}{$81-176$} & \multicolumn{3}{|c|}{331} & \multicolumn{3}{|c|}{008} & \multicolumn{3}{|c|}{019} & \multicolumn{3}{|c|}{108} & \multicolumn{3}{|c|}{434} & \multicolumn{3}{|c|}{506} \\
\hline & $\mathrm{RT}$ & 37 & 4 & $\mathrm{RT}$ & 37 & 42 & RT & 37 & 42 & RT & 37 & 42 & $\overline{\mathrm{RT}}$ & 37 & 42 & $\mathrm{RT}$ & 37 & 42 & RT & 37 & 42 & RT & 37 & 42 & $\mathrm{RT}$ & 37 & 42 & $\overline{\mathrm{RT}}$ & 37 & 42 & $\mathrm{RT}$ & 37 & 42 & $\overline{\mathrm{RT}}$ & 37 & 42 \\
\hline $1 \mathrm{~A}$ & + & + & + & + & + & + & + & + & + & + & + & + & + & + & + & + & + & + & + & + & + & + & + & + & + & + & + & + & + & + & + & + & + & + & + & + \\
\hline $1 \mathrm{~B}$ & + & + & + & + & + & + & + & + & + & + & + & + & + & + & + & + & + & + & - & - & - & + & + & + & + & + & + & + & + & + & + & + & + & + & + & + \\
\hline $1 C$ & + & + & + & + & + & + & + & + & + & + & + & + & + & + & + & + & + & + & - & - & - & + & + & + & + & + & + & + & + & + & + & + & + & + & + & + \\
\hline $1 \mathrm{D}$ & + & + & + & + & + & + & + & + & + & + & + & + & + & + & + & + & + & + & + & + & + & + & + & + & + & + & + & + & + & + & + & + & + & + & + & + \\
\hline $1 \mathrm{E}$ & + & + & + & + & + & + & + & + & + & + & + & + & + & + & + & + & + & + & + & + & + & + & + & + & + & + & + & + & + & + & + & + & + & + & + & + \\
\hline $1 \mathrm{~F}$ & + & + & + & + & + & + & + & + & + & - & - & - & + & + & + & + & + & + & + & + & + & + & + & + & + & + & + & + & + & + & + & + & + & + & + & + \\
\hline $1 \mathrm{G}$ & + & + & + & + & + & + & + & + & + & + & + & + & + & + & + & + & + & + & + & + & + & + & + & + & + & + & + & + & + & + & + & + & + & + & + & + \\
\hline $1 \mathrm{H}$ & + & + & + & + & + & + & + & + & + & + & + & + & + & + & + & + & + & + & + & + & + & + & + & + & + & + & + & + & + & + & + & + & + & + & + & + \\
\hline 11 & + & + & + & + & + & + & + & + & + & + & + & + & + & + & + & + & + & + & - & - & - & + & + & + & + & + & + & + & + & + & + & + & + & + & + & + \\
\hline $1 \mathrm{~J}$ & + & + & + & + & + & + & + & + & + & + & + & + & + & + & + & + & + & + & - & - & - & + & + & + & + & + & + & + & + & + & + & + & + & + & + & + \\
\hline $1 \mathrm{~K}$ & + & + & + & + & + & + & + & + & + & - & - & - & + & + & + & + & + & + & - & - & - & + & + & + & + & + & + & + & + & + & + & + & + & + & + & + \\
\hline $1 \mathrm{~L}$ & + & + & + & + & + & + & + & + & + & + & + & + & + & + & + & + & + & + & - & - & - & + & + & + & + & + & + & + & + & + & + & + & + & + & + & + \\
\hline $1 \mathrm{M}$ & + & + & + & + & + & + & + & + & + & + & + & + & + & + & + & + & + & + & - & - & - & + & + & + & + & + & + & + & + & + & + & + & + & + & + & + \\
\hline $1 \mathrm{~N}$ & + & + & + & + & + & + & + & + & + & + & + & + & + & + & + & + & + & + & + & + & + & + & + & + & + & + & + & + & + & + & + & + & + & + & + & + \\
\hline 10 & + & + & + & + & + & + & + & + & + & + & + & + & + & + & + & + & + & + & + & + & + & + & + & + & + & + & + & + & + & + & + & + & + & + & + & + \\
\hline $1 \mathrm{P}$ & + & + & + & + & + & + & + & + & + & + & + & + & + & + & + & + & + & + & + & + & + & + & + & + & + & + & + & + & + & + & + & + & + & + & + & + \\
\hline $2 \mathrm{~A}$ & + & + & + & + & + & + & + & + & + & + & + & + & + & + & + & + & + & + & + & + & + & $T$ & + & + & + & + & + & + & + & + & + & + & + & + & + & + \\
\hline $2 \mathrm{~B}$ & + & + & + & + & + & + & + & + & + & + & + & + & + & + & + & + & + & + & + & + & + & + & + & + & + & + & + & + & + & + & + & + & + & + & + & + \\
\hline $2 C$ & + & + & + & + & + & + & + & + & + & + & + & + & + & + & + & + & + & + & - & - & - & + & + & + & + & + & + & + & + & + & + & + & + & + & + & + \\
\hline $2 \mathrm{D}$ & + & + & + & + & + & + & + & + & + & + & + & + & + & + & + & + & + & + & - & - & - & + & + & + & + & + & + & + & + & + & + & + & + & + & + & + \\
\hline $2 \mathrm{E}$ & + & + & + & + & + & + & + & + & + & + & + & + & + & + & + & + & + & + & + & + & + & + & + & + & + & + & + & + & + & + & + & + & + & + & + & + \\
\hline $2 F$ & + & + & + & + & + & + & + & + & + & + & + & + & + & + & + & + & + & + & + & + & + & + & + & + & + & + & + & + & + & + & + & + & + & + & + & + \\
\hline $2 \mathrm{G}$ & + & + & + & + & + & + & + & + & + & + & + & + & + & + & + & + & + & + & + & + & + & + & + & + & + & + & + & + & + & + & + & + & + & + & + & + \\
\hline $2 \mathrm{H}$ & + & + & + & + & + & + & + & + & + & + & + & + & + & + & + & + & + & + & + & + & + & + & + & + & + & + & + & + & + & + & + & + & + & + & + & + \\
\hline
\end{tabular}


Table 2 Glucosamine and mannose binding from the glycan array analysis of twelve C. jejuni strains

\begin{tabular}{|c|c|c|c|c|c|c|c|c|c|c|c|c|c|c|c|c|c|c|c|c|c|c|c|c|c|c|c|c|c|c|c|c|c|c|c|c|}
\hline \multirow[t]{3}{*}{ Glycan ID } & \multicolumn{18}{|c|}{ Human } & \multicolumn{18}{|c|}{ Chicken } \\
\hline & \multicolumn{3}{|c|}{11168} & \multicolumn{3}{|c|}{351} & \multicolumn{3}{|c|}{375} & \multicolumn{3}{|c|}{520} & \multicolumn{3}{|c|}{81116} & \multicolumn{3}{|c|}{$81-176$} & \multicolumn{3}{|c|}{331} & \multicolumn{3}{|c|}{008} & \multicolumn{3}{|c|}{019} & \multicolumn{3}{|c|}{108} & \multicolumn{3}{|c|}{434} & \multicolumn{3}{|c|}{506} \\
\hline & RT & 37 & 42 & RT & 37 & 42 & RT & 37 & 42 & RT & 37 & 42 & RT & 37 & 42 & RT & 37 & 42 & RT & 37 & 42 & RT & 37 & 42 & $\mathrm{RT}$ & 37 & 42 & $\mathrm{RT}$ & 37 & 42 & $\mathrm{RT}$ & 37 & 42 & $\overline{\mathrm{RT}}$ & 37 & 42 \\
\hline $4 \mathrm{~A}$ & - & - & - & + & - & - & - & - & - & - & - & - & + & + & + & - & - & - & + & + & + & - & - & - & - & - & - & - & - & - & - & - & - & - & - & - \\
\hline $4 \mathrm{~B}$ & - & - & - & + & - & - & - & - & - & - & - & - & + & + & + & - & - & - & + & + & + & - & - & - & - & - & - & - & - & - & - & - & - & - & - & - \\
\hline $4 C$ & - & - & - & + & - & - & - & - & - & - & - & - & + & + & + & - & - & - & - & - & - & + & + & + & - & - & - & - & - & - & - & - & - & + & + & + \\
\hline $4 \mathrm{D}$ & - & - & - & + & + & + & + & + & + & + & + & + & + & + & + & + & + & + & - & - & - & + & + & + & - & - & - & - & - & - & + & + & + & + & + & + \\
\hline $4 \mathrm{E}$ & - & - & - & + & + & + & + & + & + & + & + & + & + & + & + & + & + & + & - & - & - & + & + & + & - & - & - & - & - & - & + & + & + & + & + & + \\
\hline $5 \mathrm{~A}$ & + & + & + & + & + & + & + & + & + & + & + & + & + & + & + & + & + & + & - & - & - & + & + & + & + & + & + & + & + & + & + & + & + & + & + & + \\
\hline $5 B$ & + & + & + & + & + & + & + & + & + & + & + & + & + & + & + & + & + & + & - & - & - & + & + & + & + & + & + & + & + & + & + & + & + & + & + & + \\
\hline $5 C$ & - & - & - & + & - & - & + & - & - & + & - & - & + & + & + & + & - & - & + & + & + & + & - & - & + & - & - & + & - & - & - & - & - & - & - & - \\
\hline $5 \mathrm{D}$ & - & - & - & + & - & - & + & - & - & + & - & - & + & + & + & + & - & - & - & - & - & + & - & - & + & - & - & + & - & - & - & - & - & - & - & - \\
\hline $5 \mathrm{E}$ & + & - & - & + & - & - & + & - & - & + & - & - & + & + & + & + & - & - & + & + & + & + & - & - & + & - & - & + & - & - & + & - & - & + & - & - \\
\hline $5 \mathrm{~F}$ & + & - & - & + & - & - & + & - & - & + & - & - & + & + & + & + & - & - & + & + & + & + & - & - & + & - & - & + & - & - & + & - & - & + & - & - \\
\hline $5 G$ & + & - & - & + & + & + & + & - & - & + & - & - & + & + & + & + & - & - & + & + & + & + & - & - & + & - & - & + & + & + & + & + & + & + & + & + \\
\hline $5 \mathrm{H}$ & + & - & - & + & + & + & + & - & - & + & - & - & + & + & + & + & - & - & + & + & + & + & - & - & + & - & - & + & + & + & + & + & + & + & + & + \\
\hline
\end{tabular}
(4A GlCNAC $\beta 1$ ( mannose containing GICNAC structures (5A GICNAC $31-2$ Man; $5 B$ GICNAC $31-2$ Mana1-6(GICNAC $\beta 1-2$ Mana1-3)Man), while 5C-5H are mannose of differing linkages and branch size (5C Mana1-2Man; 5D Mana1-3Man; 5E Mana1-4Man; 5 F Mana1-6Man; 5G Mana1-6(Mana1-3)Man; 5H Mana1-6(Mana1-3)Mana1-6(Mana1-3)Man). Please see Additional file 1: Table S1 for full list of glycan names and structures). 
Table 3 Binding of sialylated structures from the glycan array analysis of twelve C. jejuni strains

\begin{tabular}{|c|c|c|c|c|c|c|c|c|c|c|c|c|c|c|c|c|c|c|c|c|c|c|c|c|c|c|c|c|c|c|c|c|c|c|c|c|}
\hline \multirow[t]{3}{*}{ Glycan ID } & \multicolumn{18}{|c|}{ Human } & \multicolumn{18}{|c|}{ Chicken } \\
\hline & \multicolumn{3}{|c|}{11168} & \multicolumn{3}{|c|}{351} & \multicolumn{3}{|c|}{375} & \multicolumn{3}{|c|}{520} & \multicolumn{3}{|c|}{81116} & \multicolumn{3}{|c|}{$81-176$} & \multicolumn{3}{|c|}{331} & \multicolumn{3}{|c|}{008} & \multicolumn{3}{|c|}{019} & \multicolumn{3}{|c|}{108} & \multicolumn{3}{|c|}{434} & \multicolumn{3}{|c|}{506} \\
\hline & $\overline{R T}$ & 37 & $\overline{42}$ & $\overline{\mathrm{RT}}$ & 37 & $\overline{42}$ & $\overline{\mathrm{RT}}$ & 37 & $\overline{42}$ & $\overline{R T}$ & 37 & $\overline{42}$ & $\overline{R T}$ & 37 & $\overline{42}$ & $\overline{\mathrm{RT}}$ & 37 & $\overline{42}$ & $\overline{\mathrm{RT}}$ & 37 & $\overline{42}$ & $\overline{\mathrm{RT}}$ & 37 & $\overline{42}$ & $\overline{\mathrm{RT}}$ & 37 & $\overline{42}$ & $\overline{\mathrm{RT}}$ & 37 & $\overline{42}$ & $\overline{\mathrm{RT}}$ & 37 & $\overline{42}$ & $\overline{\mathrm{RT}}$ & 37 & 42 \\
\hline $10 \mathrm{~A}$ & + & - & - & + & + & + & + & + & + & + & + & + & + & - & - & + & - & - & + & + & + & + & - & - & + & - & - & + & - & - & + & + & + & + & + & + \\
\hline $10 \mathrm{~B}$ & + & - & - & + & + & + & + & + & + & + & + & + & + & - & - & + & - & - & + & + & + & + & - & - & + & - & - & + & - & - & + & + & + & + & + & + \\
\hline $10 C$ & + & - & - & + & - & - & + & - & - & + & - & - & + & + & + & + & - & - & + & - & - & + & - & - & + & - & - & + & - & - & + & - & - & + & - & - \\
\hline $10 \mathrm{D}$ & + & + & + & + & + & + & + & + & + & + & + & + & + & + & + & + & + & + & + & + & + & + & + & + & + & + & + & + & + & + & + & + & + & + & + & + \\
\hline $10 \mathrm{~K}$ & + & - & - & + & - & - & + & - & - & + & - & - & + & + & + & + & - & - & + & - & - & + & - & - & + & - & - & + & - & - & + & - & - & + & - & - \\
\hline $10 \mathrm{~L}$ & + & - & - & + & - & - & + & - & - & + & - & - & + & - & - & + & - & - & + & - & - & + & - & - & + & - & - & + & - & - & + & - & - & + & - & - \\
\hline $10 \mathrm{M}$ & + & - & - & + & - & - & + & - & - & + & - & - & + & - & - & + & - & - & + & - & - & + & - & - & + & - & - & + & - & - & + & - & - & + & - & - \\
\hline $10 \mathrm{~N}$ & + & - & - & + & - & - & + & - & - & + & - & - & + & - & - & + & - & - & + & - & - & + & - & - & + & - & - & + & - & - & + & - & - & + & - & - \\
\hline 100 & + & - & - & + & - & - & + & - & - & + & - & - & + & - & - & + & - & - & + & - & - & + & - & - & + & - & - & + & - & - & + & - & - & + & - & - \\
\hline $10 \mathrm{P}$ & + & - & - & + & - & - & + & - & - & + & - & - & + & - & - & + & - & - & + & - & - & + & - & - & + & - & - & + & - & - & + & - & - & + & - & - \\
\hline $11 \mathrm{~A}$ & + & - & - & + & - & - & + & - & - & + & - & - & + & - & - & + & - & - & + & - & - & + & - & - & + & - & - & + & - & - & + & - & - & + & - & - \\
\hline $11 \mathrm{~B}$ & + & - & - & + & - & - & + & - & - & + & - & - & + & - & - & + & - & - & + & - & - & + & - & - & + & - & - & + & - & - & + & - & - & + & - & - \\
\hline $11 \mathrm{C}$ & + & - & - & + & - & - & + & - & - & + & - & - & + & - & - & + & - & - & + & - & - & + & - & - & + & - & - & + & - & - & + & - & - & + & - & - \\
\hline $11 \mathrm{D}$ & + & - & - & + & - & - & + & - & - & + & + & + & + & - & - & + & - & - & + & - & - & + & - & - & + & - & - & + & - & - & + & - & - & + & - & - \\
\hline
\end{tabular}

Each of the strains were analysed at room temperature (left), $37^{\circ} \mathrm{C}$ (middle) and $42^{\circ} \mathrm{C}$ (right). Binding +; No binding -. See Additional file 1: Table S1 for full list and structures of glycans. 10A Neu5Aca2-3Gal $31-3($ Fuca1-

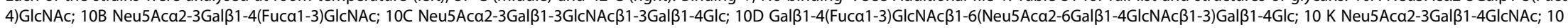

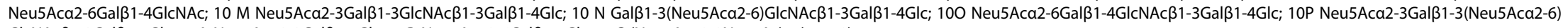
GlcNAc $\beta 1-3 G a \mid \beta 1-4 G l c ; 11 A$ Neu5Aca2-3Galß1-4Glc; 11B Neu5Aca2-6Gal $\beta 1-4 G l c ; 11 C$ (Neu5Aca2-8Neu5Ac)n $(n<50) ;$

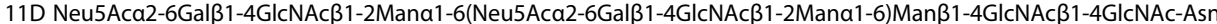


Table 4 Binding of GAG and GAG related structures from the glycan array analysis of twelve C. jejuni strains

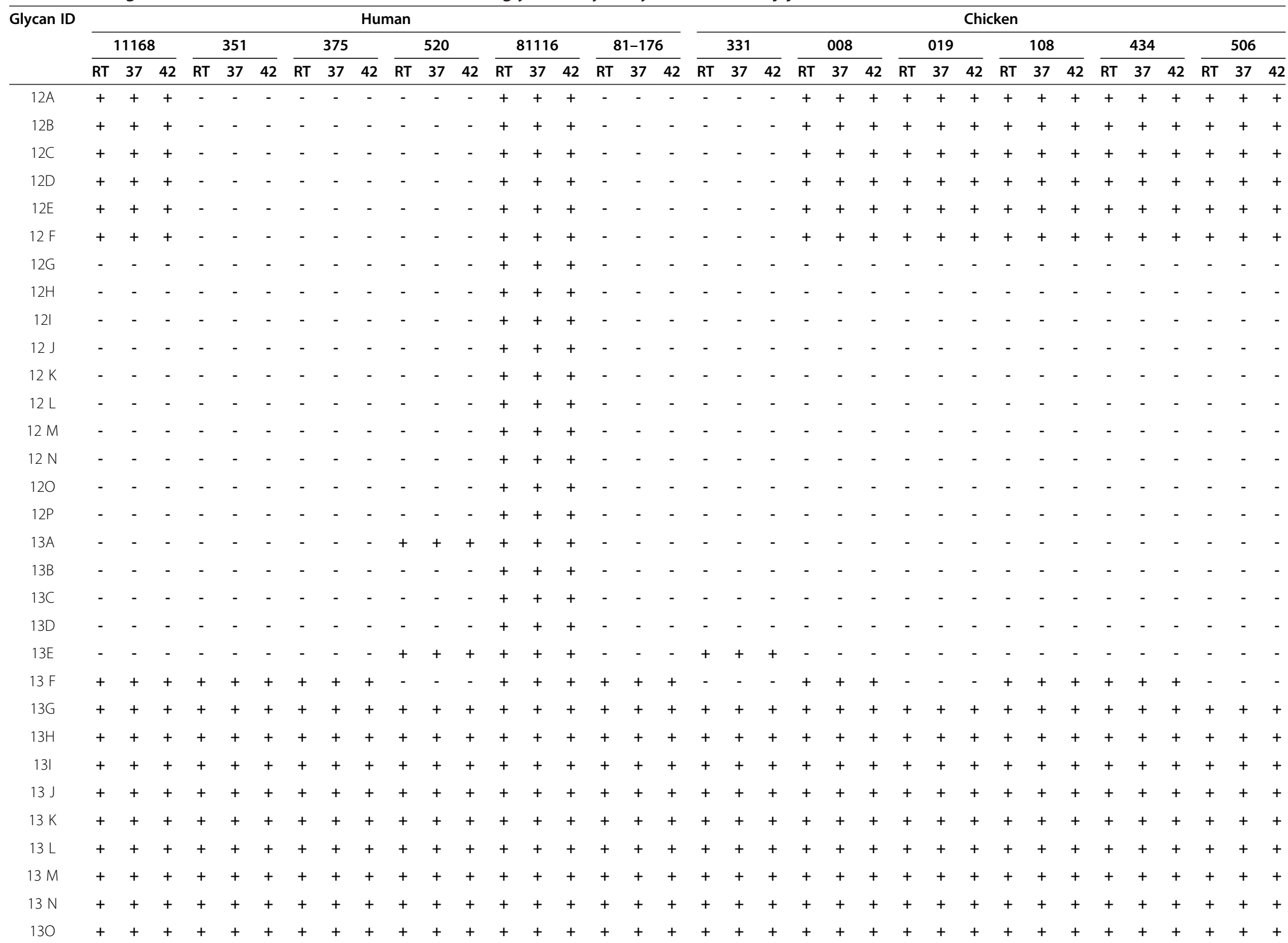


Table 4 Binding of GAG and GAG related structures from the glycan array analysis of twelve C. jejuni strains (Continued)

\begin{tabular}{|c|c|c|c|c|c|c|c|c|c|c|c|c|c|c|c|c|c|c|c|c|c|c|c|c|c|c|c|c|c|c|c|c|c|c|c|c|}
\hline 13P & & & & $T$ & + & + & + & + & + & + & + & + & + & + & + & + & $T$ & $T$ & + & + & + & + & + & + & + & + & + & + & $T$ & $T$ & $T$ & $T$ & + & + & + & \\
\hline $4 A$ & & + & + & $\mathrm{T}$ & ${ }^{\top}$ & I & + & + & + & + & 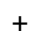 & + & 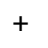 & + & + & 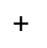 & + & + & + & + & . & . & 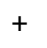 & & & & & & & & & & & & & \\
\hline $14 \mathrm{~B}$ & & 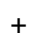 & 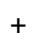 & + & + & 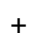 & 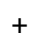 & + & + & + & + & + & 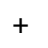 & 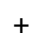 & 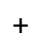 & 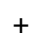 & 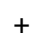 & 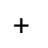 & 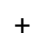 & 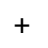 & 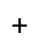 & 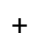 & 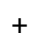 & & 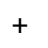 & 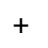 & 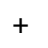 & & & 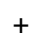 & & & & & & \\
\hline $14 C$ & + & $T$ & $T$ & $T$ & $T$ & $T$ & + & + & + & + & + & + & + & + & + & + & + & + & + & + & + & + & + & & & & & & & & & & & & & \\
\hline AD & + & + & + & + & + & + & + & + & + & + & + & + & + & + & + & + & + & + & + & + & + & + & + & + & 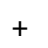 & 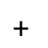 & 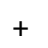 & 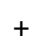 & + & + & r & $r$ & r & 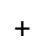 & t & \\
\hline $14 \mathrm{E}$ & + & + & + & + & + & + & + & + & + & + & + & + & + & + & + & + & + & + & + & + & + & + & + & + & + & + & + & + & + & + & + & + & + & + & + & 1 \\
\hline $4 \mathrm{~F}$ & $T$ & + & + & + & + & + & + & + & + & + & + & + & + & + & + & + & + & + & + & + & + & + & + & + & + & + & + & + & + & + & & + & + & + & + & \\
\hline $14 \mathrm{G}$ & + & + & + & + & + & + & + & + & + & + & + & + & + & + & + & + & + & + & + & + & + & + & + & + & + & + & + & r & . & . & . & r & 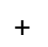 & 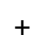 & r & \\
\hline $14 \mathrm{H}$ & + & + & + & + & + & + & + & + & + & + & + & + & + & + & + & + & + & + & + & + & + & + & + & + & 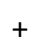 & 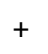 & 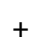 & 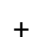 & 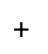 & 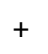 & 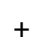 & + & + & + & + & \\
\hline 14 & + & + & + & + & + & + & + & + & + & + & + & + & + & + & + & + & + & + & + & + & $T^{T}$ & 1 & r & r & 1 & r & r & r & r & $r^{\prime}$ & 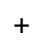 & $T$ & $T$ & $\tau$ & 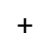 & + \\
\hline
\end{tabular}

Each of the strains were analysed at room temperature (left), $37^{\circ} \mathrm{C}$ (middle) and $42^{\circ} \mathrm{C}$ (right). Binding +; No binding -. 12A-12 F are Carrageenan repeats; $12 \mathrm{G}-13 \mathrm{E}$ are digested Glycoaminoglycans in their most basic

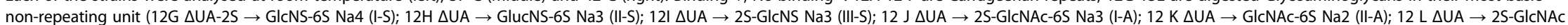

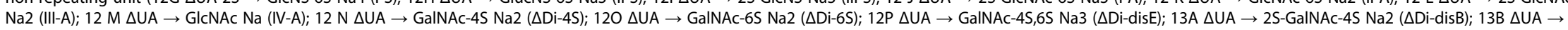

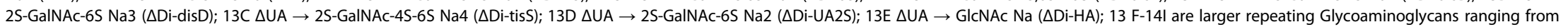

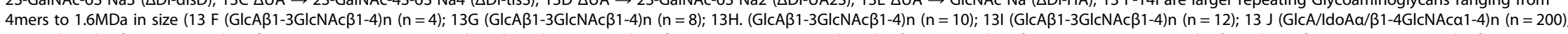

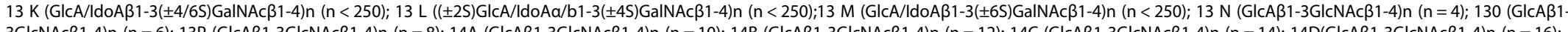

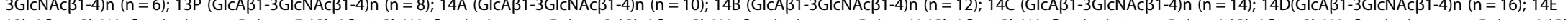

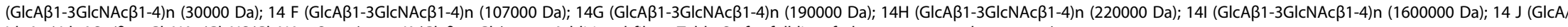

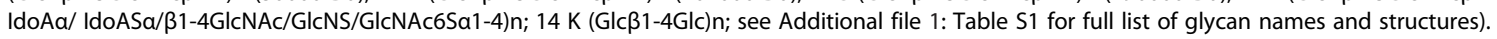


isolates, only C. jejuni 11168 and 81116 bound to the carageenan structures. C. jejuni 81116 was the only strain that bound with any consistency to the enzymatically digested GAG disaccharide fragments (Table 4; 12G-13H). However, all strains of C. jejuni tested bound to hyaluronin, chondrotin, heparin and dermatin.

\section{Lectin and glycan competition adherence assays}

As previously shown with C. jejuni 11168, lectins that recognise structures similar or identical to those recognised by $C$. jejuni, can be used to inhibit adherence to the surface of Caco-2 cells [3]. For the adherence inhibition assays, using both lectins and free glycans, C. jejuni was grown at $37^{\circ} \mathrm{C}$ in a microaerobic environment, mimicking one of the growth conditions used in glycan arrays assays. Two lectins were tested; ConA (mannose binding lectin) and UEA-I (fucose binding lectin).

As predicted from the array results, ConA had the greatest inhibitory effects on the adherence of $C$. jejuni 81116 and 331 with reductions of more than $70 \%$, no significant difference was observed for the other strains tested (Figure 1A). UEA-I resulted in significant reduction in adherence for all strains tested but did not affect the adherence of the control E. coli DH5a strain (Figure 1B).

Free glycans were also tested on the adherence of two C. jejuni strains; the clinical isolate 11168 and the chicken isolate 331. Using $100 \mu \mathrm{M}$ of free blood group antigens, A blood group trisaccharide (glycan $7 \mathrm{~K}$ on the array) and the $\mathrm{H}$ disaccharide (O-blood group antigen; glycan $7 \mathrm{~F}$ on the array), resulted in the significant decrease of adherence of both $C$. jejuni $11168(\mathrm{P}<0.05)$ and $331(\mathrm{P}<0.05)$ to Caco-2 cells (Figure $1 \mathrm{C})$. Free mannose $(\alpha 1-2$ Mannobiose at $100 \mu \mathrm{M}$; glycan $5 \mathrm{C}$ on the array) had no effect on the binding of $C$. jejuni 11168 to Caco-2 cells but did significantly reduce the adherence of C. jejuni 331 ( $\mathrm{P}<0.05$; Figure $1 \mathrm{C})$. This result is in agreement with the array data, with both strains binding blood group antigens but only $C$. jejuni 331 recognising mannose under the condition tested (Table 2).

\section{Discussion}

All C. jejuni strains tested in this study showed remarkable similarity for the general types of glycan structures that were recognised. Looking globally at the total array, C. jejuni behaves as a species with little variation, each strain bound to both $\alpha$ and $\beta$ galactose, terminal and subterminal fucosylated structures and to a subset of glycoaminoglycans at all conditions tested. All strains also exhibited binding to a broader range of glycans when placed under environmental stress. Only chitin, a common insect and crustacean glycan, showed major differences when viewed from a global perspective, with
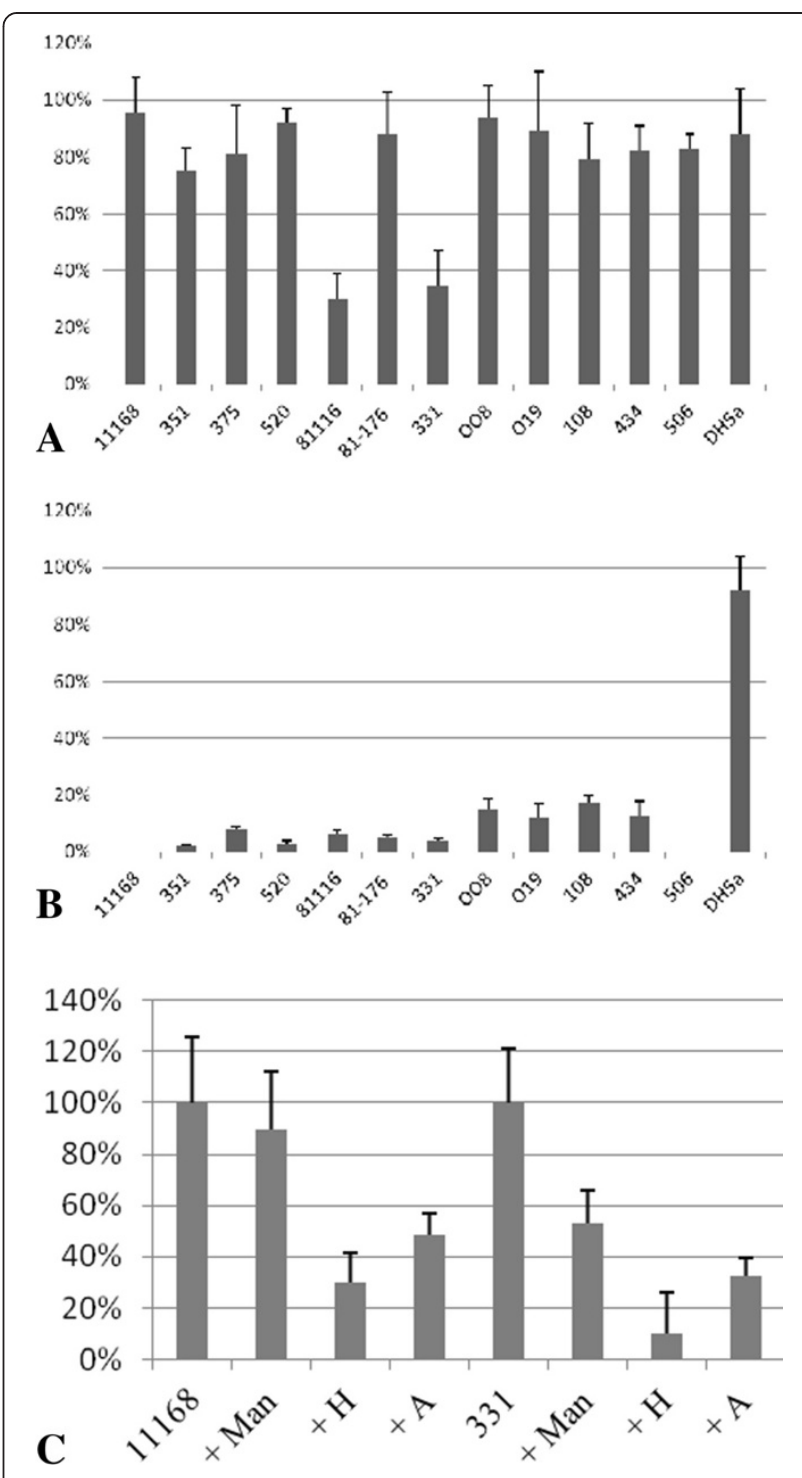

Figure 1 Lectin and free glycan competition assays. Comparison between normal adherence (100\%) and inhibition with lectin or glycan pre-treatment. The smaller the bar the less C. jejuni adhered in the presence of the lectin/glycan. A. ConA competition of C. jejuni adherence to Caco-2 cells; B. UEA-I competition of C. jejuni adherence to Caco-2 cells. C. Competion assays with free glycans with C. jejuni 11168 and 331 adhering to Caco-2 cells.

one strain, $C$. jejuni 11168 , failing to recognise any chitin molecule. No major difference was observed between $C$. jejuni strains isolated from different hosts.

The possibility of galactose and fucose being involved in the persistent colonisation of C. jejuni $[3,4]$ is supported by the interactions observed in this study. All twelve strains, whether isolated from avian or clinical sources, bound broadly to uncapped galactose structures and fucosylated structures. These results were confirmed by inhibition of adherence to cells blocked by competing C. jejuni adherence with UEA-I. 
Of the strains tested only one chicken isolate (331) and one clinical isolate (520) showed variability in the galactose structures bound. Of interest is the broad specificity of all the $C$. jejuni strains for galactose and fucosylated structures. Only strain, C. jejuni 520, showed binding differences based on linkage specificity with Gal/31-3GalNAc (asialo-GM1 1 F) and terminal $\alpha-1-4$ linked di-galactose $(1 \mathrm{~K})$ glycan structures not being recognised.

The fact that $C$. jejuni recognises a broad range of both $\alpha$ and $\beta$ linked galactose may offer some explanation for such a broad host range, as might the lack of specificity for linkage and position of fucose in fucosylated structures. $\alpha$ linked galactose are not common in humans but are common in many other mammals and avian species [13-17]. Some strains of $C$. jejuni are known to produce the Pantigen, a terminal $\alpha$-linked galactose, as a part of their LOS structure to mimic the glycans of potential avian and non-human mammalian hosts $[13,18]$. $\beta$-linked galactose structures are common to all animals known to be infected with $C$. jejuni. The fact that $C$. jejuni recognises both $\alpha$ and $\beta$ linked galactose indicates either a broad specificity galactose binding lectin or two or more lectins with restricted specificity. As binding to these different galactose structures is not preferential under any condition tested, it is likely that a single yet to be identified broad specificity glactose binding lectin is expressed by $C$. jejuni.

Fucose is a known chemoattractant of $C$. jejuni but the binding observed in our glycan array analysis is unlikely to be related to the periplasmic receptors for chemotaxis. Fucose surface expression in humans is dependent on a range of fucosyltransferases that can be differentially expressed both throughout tissues and between individuals resulting in differential fucosylation between tissue types or differential fucosylation of the same tissue types when comparing two nonrelated individuals. As C. jejuni has no preference for linkage or location it is likely that either the same protein that recognises galactose is binding fucosylated structures but ignoring the presence of fucose or that $C$. jejuni has a broad specificity fucose binding lectin.

Binding to $\mathrm{N}$-acetylglucosamine structures was differential between strains with three strains not recognising GlcNAc structures at all (C. jejuni 11168, 019 and 108). Typically among strains that did recognise GlcNAc structures the longer repeats were preferred. Only C. jejuni 331 (under all conditions), 81116 (under all conditions) and 351 (under environmental conditions) recognised the short repeats. Chitin a common glycoconjugate found in insects and crustaceans is comprised of repeating GlcNAc residues. It is possible that $C$. jejuni strains that recognise GlcNAc structures may use insects as vectors as described by Hald et al. [19], or that strains with GlcNAc recognition can better infect crustaceans to survive and propagate in fresh water ponds and streams $[19,20]$. Chitin recognition may therefore be important for environmental survival and spread, also offering advantages for re-infection of more preferred avian or mammalian hosts.

In line with previously reported data [3], mannose was recognised more often after environmental stress by most of the C. jejuni strains tested. C. jejuni 331 and 81116 were the only strains to recognise a wide variety of mannose structures under all growth/maintenance conditions. Several other strains, more common to the chicken isolates tested (Human isolate: C. jejuni 351; Chicken isolates: C. jejuni 108, 434 and 506), also recognised some of the branched mannose structures under all conditions tested. Branched mannose is far more common in complex N-linked glycans found on many different cell surface proteins. These branched mannose structures are typically capped by other sugars including Glc/GlcNAc, Gal/ GalNAc and sialic acid implying that either these interactions are through subterminal binding proteins that can recognise capped structures or are not biologically relevant to infection/colonisation. From the binding profile of C. jejuni to the complex sialylated structure, 11D, it appears in all cases but $C$. jejuni 108 that subterminal recognition of mannose in complex N-linked glycans can be ruled out.

Similar to $C$. jejuni binding to mannose, sialic acid recognition was only observed following a period of environmental stress, with all the $C$. jejuni strains tested exhibiting significantly more binding to sialylated glycans when maintained under normal atmosphere and at room temperature. This indicates that an adhesion/lectin able to bind sialylated glycans is regulated by the exposure of $C$. jejuni to environmental stress. As yet, no such protein has been elucidated in C. jejuni. Sialic acid is a common glycan present on multiple cell types and is typically the terminal sugar presented. In the intestines MUC1 is the most heavily sialylated protein present, however, MUC1 acts as a decoy receptor for bacteria and other viral and microbial infecting agents [10]. When MUC1 is bound by pathogens it is released from the cell surface and allows the pathogen to be excreted into the environment through the lumen [10]. A number of pathogens, including C. jejuni, are more infectious, have a lower infectious dose or get into deeper tissues faster when administered to $\mathrm{MUC1}^{-/-}$mice [10].

Of the few sialylated structures that were bound more broadly by C. jejuni, 10A (C. jejuni strains 351, 375, 520, 331, 434, 506), 10B (C. jejuni strains 351, 375, 520, 331, $434,506)$ and 10D (all strains tested), are all fucosylated, indicating that the binding to these glycans may be more due to fucose than to sialic acid. C. jejuni 81116, once again, recognised a wider variety of sialic acid containing structures than the other $C$. jejuni strains tested, binding to $\alpha 2-3$ linked sialylactosamine structures. C. jejuni 81116 has a vastly different cell surface glycosylation profile than other $C$. jejuni producing larger non-sialylated LPS like 
molecule rather than the traditional LOS seen for other $C$. jejuni [21]. It may be interesting to speculate that surface glycosylation can play a role in the inhibition of the binding of $C$. jejuni to sialylated glycans, particularly through charge-charge repulsion. Sialic acid is a negatively charged sugar and C. jejuni strains such as 11168 are known to have surface glycosylation that contains sialic acid [22,23]. Of the strains that bound to sialyllewis structures (10A and B), we have recently shown that, C. jejuni 351, 375 and 331, do not have surface sialylation [24], indicating these strains may be able to recognise the underlying fucose. We are yet to confirm the sialylation levels of $C$. jejuni strains 434 and 506. C. jejuni 520 seems to be a special case as the LOS it produces appears to be very heterogenous [24]. We have shown using lectin array and surface plasmon resonance that a proportion of the LOS produced by this strain is completely non-sialylated at all growth conditions tested [24]. It is therefore possible that sufficient $C$. jejuni 520 was present in the assay with low or no surface sialylation allowing for recognition of the underlying branched fucose.

Glycoaminoglycan binding by $C$. jejuni on glycan arrays has not previously been reported. C. jejuni in general preferred larger GAG fragments, with the most consistent binding observed to full length GAGs of up to $1.6 \mathrm{MDa}$. GAGs are common extracellular matrix components and are expressed in on the surface of a broad range of cells [25-27]. GAGs are also known to associate with known cell surface targets of $C$. jejuni including fibronectin [25-27]. Once more 81116 had the broadest recognition for GAG and related structures recognising all the structures present on our array.

The non-invasive $C$. jejuni strain 331 had a preference for longer, branched galactose structures and was less likely to associate with disaccharides or terminal $\mathrm{N}$ Acetylgalactosamine structures. This is of interest as $C$. jejuni 331 is known to be a strong chicken coloniser, capable of out competing other C. jejuni strains in coinfection studies and has been proposed as a potential non-virulent bioreplacement bacteria $[28,29]$. It is possible that the lack of binding to disaccharide and small sugar subunits by $C$. jejuni 331 may offer a competitive advantage, allowing 331 to better colonise the intestinal crypts by ignoring smaller sugars in the lumen. Monoand di-saccharides are common products from the activity of glycosidases in the intestinal tract of animals. This makes mono- and di-saccharides potential decoy receptors for $C$. jejuni in the chicken gut and as such, bacteria that do not bind to smaller sugars would potentially have a competitive advantage.

\section{Conclusions}

The conclusions drawn from the initial screening of C. jejuni 11168 on our glycan array [3] have in the main been confirmed by the screening of additional strains. Sialic acid and mannose still appear to be the general structures recognised after environmental stress, appearing to be important for initial host pathogen interactions. Galactose and fucose structures still appear to be crucial for the persistence of infection. Little difference is seen between the isolates from clinical or chicken hosts, with the exception of carageenan and branched mannose binding, with both more likely to be recognised by chicken isolates than those isolated from humans. This study increases the understanding of C. jejuni glycan recognition and provides a model for the study of complex glycan recognition from a number of other yet to be screened bacterial species.

\section{Methods}

\section{Bacterial strains and growth conditions}

The strains used in this study can be found in Table 5 . Bacteria were grown as previously described [3].

\section{Glycan arrays}

Glycan arrays were prepared and performed as previously described by Day et al. [3] with slight modification to the preparation of the slides as outlined by Hartley-Tassell et al. [30] using the glycan library described in Arndt et al. $[3,30,31]$. See Additional file 1: Table S1 for full list and structures of glycans. The arrays were scanned by a ProScan Array scanner at $488 / 520 \mathrm{~nm}$ and the results analysed by ScanArray Express software program. Binding was defined as a value greater than 1 fold increase above mean background relative fluorescence units (RFU). The mean background was calculated from the average background of empty spots on the array plus three standard deviations. Statistical analysis of the data was performed

Table 5 Bacterial strains used in this study

\begin{tabular}{ccc}
\hline Strain & Invasive & Source \\
\hline Human & $+/-$ & \\
11168 & + & D. Newell \\
351 & + & RMIT \\
375 & + & RMIT \\
520 & + & RMIT \\
81116 & + & D. Newell \\
$81-176$ & + & J. G. Fox \\
Chicken & & \\
331 & - & RMIT \\
8 & + & RMIT \\
19 & + & RMIT \\
108 & + & RMIT \\
434 & + & RMIT \\
506 & + & RMIT \\
\hline
\end{tabular}


by a Student's t-test with a confidence level of $99.99 \%$ $(\mathrm{p} \leq 0.0001)$. All arrays were performed in triplicate with a total of 12 data points for each glycan tested.

\section{Lectin competition adherence assays}

Adherence and lectin competition assays were performed as previously described [3], however, only using C. jejuni grown at $37^{\circ} \mathrm{C}$ under micraerobic conditions. E. coli $\mathrm{DH} 5 \alpha$ cells were used as a control for the lectin competition assays to ensure that reduction in adherence was not due to steric hindrance of the lectins on the cell surface inhibiting cell binding to non-glycan targets. Lectins were used at $10 \mu \mathrm{g}$ per well. All assays were performed in triplicate.

\section{Free glycan inhibition assay}

Adherence assays were performed as previously described [3] under conditions described above. Two exemplary strains of $C$. jejuni were used for this analysis, the human clinical isolate 11168 and the chicken isolate 331. Free glycan (H-disaccharide, A-blood group antigen and $\alpha 1-2$ mannobiose) were added to the media at a final concentration of $100 \mu \mathrm{M}$ just prior to addition of the bacteria.

\section{Additional file}

Additional file 1: Table of Glycans.

\section{Competing interests}

The authors declare that they have no competing interests.

\section{Authors' contributions}

CJD conceived the experiments, performed many of the array and all the cell culture experiments and aided in the analysis of the data. CJD wrote a significant portion of the completed manuscript. GT helped perform array experimentation, aided with the glycan inhibition cell culture assays, helped analyse data and aided in the production of the manuscript. LEH-T helped performed array experimentation, helped analyse data including the establishment of the statistical template and aided in the production of the manuscript. JT helped performed array experimentation, helped analyse data and aided in the production of the manuscript. VK conceived the experiments, aided in the analysis of the data and was responsible for final edits of the completed manuscript. All authors read and approved the final manuscript.

\section{Acknowledgements}

This work was partially funded by and CJD and LHT are supported by a Queensland State Government, Department of Science, Information Technology, Innovation and the Arts Research Partnerships Program. GT is supported by a Griffith University Postgradute Research Scholarship.

Received: 29 July 2013 Accepted: 27 September 2013 Published: 14 October 2013

\section{Reference}

1. Smith DC, Lord JM, Roberts LM, Johannes L: Glycosphingolipids as toxin receptors. Semin Cell Dev Biol 2004, 15(4):397-408.

2. Lehmann F, Tiralongo $E$, Tiralongo J: Sialic acid-specific lectins: occurrence, specificity and function. Cell Mol Life Sci 2006, 63(12):1331-1354.

3. Day CJ, Tiralongo J, Hartnell RD, Logue CA, Wilson JC, von Itzstein M, Korolik $\checkmark$ : Differential carbohydrate recognition by Campylobacter jejuni strain
11168: influences of temperature and growth conditions. PLOS One 2009, 4(3):e4927.

4. Day CJ, Semchenko EA, Korolik V: Glycoconjugates play a key role in campylobacter jejuni infection: interactions between host and pathogen. Front Cell Infect Microbiol 2012, 2:9.

5. Newburg DS, Ruiz-Palacios GM, Morrow AL: Human milk glycans protect infants against enteric pathogens. Annu Rev Nutr 2005, 25:37-58.

6. Morrow AL, Ruiz-Palacios GM, Jiang X, Newburg DS: Human-milk glycans that inhibit pathogen binding protect breast-feeding infants against infectious diarrhea. J Nutr 2005, 135(5):1304-1307.

7. Yamaoka Y: Roles of Helicobacter pylori BabA in gastroduodenal pathogenesis. World J Gastroenterol 2008, 14(27):4265-4272.

8. Juge N: Microbial adhesins to gastrointestinal mucus. Trends Microbiol 2012, 20(1):30-39.

9. Mahdavi J, Sonden B, Hurtig M, Olfat FO, Forsberg L, Roche N, Angstrom J, Larsson T, Teneberg S, Karlsson KA, et al: Helicobacter pylori SabA adhesin in persistent infection and chronic inflammation. Science 2002, 297(5581):573-578.

10. McAuley JL, Linden SK, Png CW, King RM, Pennington HL, Gendler SJ, Florin TH, Hill GR, Korolik V, McGuckin MA: MUC1 cell surface mucin is a critical element of the mucosal barrier to infection. J Clin Invest 2007, 117(8):2313-2324.

11. Varki A: Multiple changes in sialic acid biology during human evolution. Glycoconj J 2009, 26(3):231-245.

12. Le Pendu J: Histo-blood group antigen and human milk oligosaccharides: genetic polymorphism and risk of infectious diseases. Adv Exp Med Biol 2004, 554:135-143.

13. Suzuki N, Laskowski M Jr, Lee YC: Phylogenetic expression of Galalpha14Gal on avian glycoproteins: glycan differentiation inscribed in the early history of modern birds. Proc Natl Acad Sci USA 2004, 101(24):9023-9028.

14. Galili U, Clark MR, Shohet SB, Buehler J, Macher BA: Evolutionary relationship between the natural anti-Gal antibody and the Gal alpha 1--3Gal epitope in primates. Proc Natl Acad Sci USA 1987, 84(5):1369-1373.

15. Yang Z, Bergstrom J, Karlsson KA: Glycoproteins with Gal alpha 4Gal are absent from human erythrocyte membranes, indicating that glycolipids are the sole carriers of blood group P activities. J Biol Chem 1994, 269(20):14620-14624.

16. Sandrin MS, McKenzie IF: Gal alpha $(1,3)$ Gal, the major xenoantigen(s) recognised in pigs by human natural antibodies. Immunol Rev 1994, 141:169-190.

17. Garratty G: Blood group antigens as tumor markers, parasitic/bacterial/ viral receptors, and their association with immunologically important proteins. Immunol Invest 1995, 24(1-2):213-232.

18. Houliston RS, Vinogradov E, Dzieciatkowska M, Li J, St Michael F, Karwaski MF, Brochu D, Jarrell HC, Parker CT, Yuki N, et al: Lipooligosaccharide of Campylobacter jejuni: similarity with multiple types of mammalian glycans beyond gangliosides. J Biol Chem 2011, 286(14):12361-12370.

19. Hald B, Skovgard H, Pedersen K, Bunkenborg H: Influxed insects as vectors for Campylobacter jejuni and Campylobacter coli in Danish broiler houses. Poult Sci 2008, 87(7):1428-1434

20. Schallenberg M, Bremer PJ, Henkel S, Launhardt A, Burns CW: Survival of Campylobacter jejuni in water: effect of grazing by the freshwater crustacean Daphnia carinata (Cladocera). Appl Environ Microbiol 2005, 71(9):5085-5088.

21. Holden KM, Gilbert M, Coloe PJ, Li J, Fry BN: The role of WlaRG, WlaTB and WlaTC in lipooligosaccharide synthesis by Campylobacter jejuni strain 81116. Microb Pathog 2012, 52(6):344-352.

22. St Michael F, Szymanski CM, Li J, Chan KH, Khieu NH, Larocque S, Wakarchuk WW, Brisson JR, Monteiro MA: The structures of the lipooligosaccharide and capsule polysaccharide of Campylobacter jejuni genome sequenced strain NCTC 11168. Eur J Biochem 2002, 269(21):5119-5136.

23. Semchenko EA, Day CJ, Wilson JC, Grice ID, Moran AP, Korolik V: Temperature-dependent phenotypic variation of Campylobacter jejuni lipooligosaccharides. BMC Microbio/ 2010, 10:305.

24. Semchenko EA, Day CJ, Moutin M, Wilson JC, Tiralongo J, Korolik V: Structural heterogeneity of terminal glycans in Campylobacter jejuni lipooligosaccharides. PLoS One 2012, 7(7):e40920.

25. Yamada KM, Kennedy DW, Kimata K, Pratt RM: Characterization of fibronectin interactions with glycosaminoglycans and identification of active proteolytic fragments. J Biol Chem 1980, 255(13):6055-6063.

26. Konkel ME, Garvis SG, Tipton SL, Anderson DE Jr, Cieplak W Jr: Identification and molecular cloning of a gene encoding a fibronectin-binding protein (CadF) from Campylobacter jejuni. Mol Microbiol 1997, 24(5):953-963. 
27. Chung CY, Erickson HP: Glycosaminoglycans modulate fibronectin matrix assembly and are essential for matrix incorporation of tenascin-C. J Cell Sci 1997, 110(Pt 12):1413-1419.

28. Calderon-Gomez LI, Hartley LE, McCormack A, Ringoir DD, Korolik V: Potential use of characterised hyper-colonising strain(s) of Campylobacter jejuni to reduce circulation of environmental strains in commercial poultry. Vet Microbiol 2009, 134(3-4):353-361.

29. Korolik V, Alderton MR, Smith SC, Chang J, Coloe PJ: Isolation and molecular analysis of colonising and non-colonising strains of Campylobacter jejuni and Campylobacter coli following experimental infection of young chickens. Vet Microbiol 1998, 60(2-4):239-249.

30. Hartley-Tassell LE, Shewell LK, Day CJ, Wilson JC, Sandhu R, Ketley JM, Korolik V: Identification and characterization of the aspartate chemosensory receptor of Campylobacter jejuni. Mol Microbiol 2010, 75(3):710-730

31. Arndt NX, Tiralongo J, Madge PD, von Itzstein M, Day CJ: Differential carbohydrate binding and cell surface glycosylation of human cancer cell lines. J Cell Biochem 2011, 112(9):2230-2240.

doi:10.1186/1471-2180-13-228

Cite this article as: Day et al: Assessment of glycan interactions of clinical and avian isolates of Campylobacter jejuni. BMC Microbiology 2013 13:228.

\section{Submit your next manuscript to BioMed Central and take full advantage of:}

- Convenient online submission

- Thorough peer review

- No space constraints or color figure charges

- Immediate publication on acceptance

- Inclusion in PubMed, CAS, Scopus and Google Scholar

- Research which is freely available for redistribution 